Tropical Journal of Pharmaceutical Research April 2010; 9 (2): 173-179

(c) Pharmacotherapy Group,

Faculty of Pharmacy, University of Benin,

Benin City, 300001 Nigeria.

All rights reserved.

Research Article

Available online at http://www.tjpr.org

\title{
Development of a Rapid Chemical Identification System (RCIS) for the Detection of Fraudulently Labelled 5-nitroimidazole Products
}

\author{
Olajide S Awofisayo ${ }^{1 \star}$, Oladoja A Awofisayo ${ }^{2}$ and Nse Eyen ${ }^{3}$ \\ ${ }^{1}$ Department of Clinical Pharmacy and Biopharmacy, ${ }^{2}$ Department of Pharmaceutical and Medicinal Chemistry, \\ Faculty of Pharmacy, University of Uyo, ${ }^{3}$ Laboratory Science Department. Akwa Ibom State Polytechnic, Nigeria.
}

\begin{abstract}
Purpose: A simple, reliable and rapid chemical identification system (RCIS) consisting of three colour reactions based on the functional groups in the molecule and two TLC methods was developed for preliminary detection of the 5-nitroimidazole drugs.

Methods: Three members of this group of drugs (tablet form) available in the Nigerian market and labelled $M_{A}$ and $M_{B}$ for metronidazole, $T_{A}$ and $T_{B}$ for tinidazole and $S$ for secnidazole, were used. The extraction of the active ingredient from the solid dosage form was performed using acetone. The reaction of the extracted drug with zinc and $1 \mathrm{M}$ hydrochloric acid at $100^{\circ} \mathrm{C}$ converted the nitroimidazole group to a characteristic primary aromatic amine. TLC methods $A$ and $B$ were carried out on $G_{254}$ plates $(5 \mathrm{~cm} \times 10 \mathrm{~cm})$ to further identify the individual members of the group. TLC method $A$ with mobile phase consisting of acetone, ethyl acetate and ammonia (100:5:1) and method $B$ with mobile phase consisting of acetone, chloroform and ammonia (100:15:1) were developed for the identification.

Results: The aromatic character of 5-nitroimidazole was highlighted in nitric acid when combined with sulphuric acid resulting in an orange colour. $40 \%$ sodium hydroxide gave the alkali-induced characteristic orange colour of aromatic amino compounds. All the samples of the various brands gave characteristic colours that distinguished the compounds of the 5-nitroimidazole group as primary or secondary nitroimidazole compounds.

Conclusion: Using the developed method, fraudulently labelled product 5-nitroimidazole antiprotozoal and antibacterial agents can now be detected in approx. 40 min with limited reagents and a simple TLC technique. The method is rugged, simple, and should be particularly handy for use in detecting substandard products of the drug in the drug distribution chain where sophisticated equipment are often not available.
\end{abstract}

Keywords: 5-nitroimidazole; Fraudulently labeled; Chemical identification system; Antiprotozoal; Antibacterial. 


\section{INTRODUCTION}

The world is confronted by the reality of large-scale counterfeit production of almost every conceivable drug $[1,2]$. It is not surprising then that drugs are produced in gross violation of good manufacturing practices (GMP) and in utter disregard of pharmacopoeia standards [2,3]. It is, therefore, desirable that deliberate and fraudulent labeling of drugs with respect to identity should be detected along the distribution chain as part of normal quality assurance processes [4]. There is the need to make available useful methods for accurately validating the authenticity and identity of available medications. This is in view of the fact that the greatest health risk faced by people of the world today is the lack of appropriate medicines for treating both common and life-threatening illnesses [4,5]. The dangers posed by counterfeit products to public health in Nigeria have resulted in control measures by the Agency for Food and Drug Administration and Control (NAFDAC) such as daily enlightenment campaigns in the media $[6,7]$. A way to complement NAFDAC's efforts against counterfeit drugs is to develop a simple and rapid method of identifying fake products [8]. The growing challenge is halting the distribution and marketing of such medicines at every level of the distribution chain $[9,10]$.

Equipment with multi-component profiling for the screening of chemicals have been documented in literature but their high cost often render unaffordable to several stakeholder along the distribution chain in several developing countries $[11,12]$. Furthermore, such equipment requires technical handling and maintenance that are beyond the capacity of those in the distribution chain [13]. A Fast Chemical Identification System (FCIS), as has been used in China since 2005, needs to be developed and compiled for the use of pharmacists and other stakeholders to include drugs such as anti-infective drugs, analgesics, cardiovascular agents, etc. FCIS is usually targeted at the molecular structure and functional groups in the drug molecule [14-16].

The 5-nitroimidazole compounds are widely used intestinal and extra-intestinal amoebicide. There has been documented evidence of the efficacy of the 5nitroimidazoles on the causative organism, Entamoeba histolytica [17]. The increase in cases of amoebiasis is said to be traceable to counterfeiting [18-20]. The 5-nitroimidazoles most commonly found in the Nigerian market are metronidazole, tinidazole and secnidazole (Fig 1).

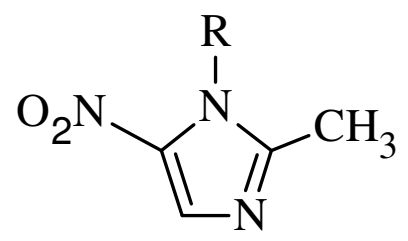

R Metronidazole $-\mathrm{CH}_{2}-\mathrm{CH}_{2} \mathrm{OH}$

2-methyl-5 nitroimidazole-1-ethanol

Tinidazole $-\mathrm{CH}_{2} \mathrm{SO}_{2} \mathrm{C}_{2} \mathrm{H}_{5}$

Ethyl [2-(2-methyl-5-nitro-1-imidazolyl)] ethyl

sulphone Secnidazole $-\mathrm{CH}_{2}-\mathrm{CH}(\mathrm{OH})-\mathrm{CH}_{3}$

$\propto$, 2-Dimethyl-5-nitro-1H-imidazole-1-ethanol

Fig 1: Chemical structure of 5-nitroimidazole compounds

\section{EXPERIMENTAL}

\section{Materials}

Branded products of metronidazole, tinidazole and secnidazole in tablet form were purchased from a registered pharmacy in Uyo, Nigeria. Free samples of these drugs were also obtained from May and Baker Plc and Cadila Pharmaceuticals Ltd, both in Lagos, Nigeria. Paracetamol tablets were similarly purchased while pure paracetamol powder was obtained free of charge from May and Baker Plc. Further details are shown in Table 1. Chloroform, acetone, methanol, ethyl acetate, bismuth subnitrate, hydrochloric acid, sodium hydroxide, sulphuric acid, nitric acid, sodium nitrate were 
bought from Nanjing Chemical Reagent Company, China, and were of analytical grade. TLC silica $\mathrm{GF}_{254}$ plates $(5 \mathrm{~cm} \times 10 \mathrm{~cm})$ were cut from $20 \mathrm{~cm} \times 30 \mathrm{~cm}$ plates (Acres Organics, Belgium).

\section{Preparation of test solution and crystals}

Tablets of each of the drugs were crushed in a mortar and reduced into fine powder, and 1 $\mathrm{g}$ of the powder was shaken thoroughly in $100 \mathrm{ml}$ of acetone in a conical flask. The drug was extracted into acetone and filtered with a filter paper. The filtrate $(50 \mathrm{ml})$ was allowed to stand at room temperature for $15 \mathrm{~min}$ to evaporate, yielding crystals. To $50 \mathrm{mg}$ of the crystals of each drug was added zinc dust $(50 \mathrm{mg})$ and $5 \mathrm{ml} 1 \mathrm{M}$ hydrochloric acid in a beaker in a boiling water bath for $5 \mathrm{~min}$. The beaker was removed and then left to stand at room temperature for $20 \mathrm{~min}$. The resulting product was dissolved in $50 \mathrm{ml}$ acetone at 50 ${ }^{\circ} \mathrm{C}$ in a water bath and filtered hot. The filtrate was again allowed to stand for $10 \mathrm{~min}$ at room temperature to allow the solvent to evaporate, leaving crystals. An amount of the crystals $(20 \mathrm{mg})$ was dissolved in $10 \mathrm{ml}$ chloroform to form the test solution. Similarly, $20 \mathrm{mg}$ of paracetamol powder was dissolved in $10 \mathrm{ml}$ acetone to form the test solution for paracetamol.

\section{Colour reactions}

Nitric acid reaction: To $1 \mathrm{ml}$ of the test solution was added three drops of $2 \mathrm{M}$ nitric acid. One drop of sodium hydroxide (40 $\% \mathrm{w} / \mathrm{v}$ ) was added after $2 \mathrm{~min}$.

Sodium nitrite reaction: $1 \mathrm{~g}$ of sodium nitrite and $1 \mathrm{ml}$ of $2 \mathrm{M}$ sulphuric acid were mixed in a test tube and cooled in ice. To $1 \mathrm{ml}$ of the test solution was added one drop of the sodium nitrite reagent.

Bismuth subnitrate reaction: Bismuth subnitrate $(20 \mathrm{mg})$ was dissolved in $3 \mathrm{ml}$ of $2 \mathrm{M}$ hydrochloric acid. This was diluted with water to $10 \mathrm{ml}, 10 \mathrm{~g}$ of potassium iodide was added. This reagent $(2 \mathrm{ml})$ was added to $1 \mathrm{ml}$ of the test solution.

\section{TLC identification}

Thin layer identification (TLC) identification was carried out on a $\mathrm{GF}_{254}$ plate $(5 \mathrm{~cm} \mathrm{x}$ $10 \mathrm{~cm})$. Mobile phase A consisted of acetone, ethyl acetate and ammonia (100:5:1, v/v) while phase $B$ consisted of acetone, chloroform and ammonia (100:15:1, v/v). Crystals obtained from the similarly treated drug products were dissolved in acetone to give a reference solution of $10 \mathrm{mg} / \mathrm{ml}$. Two

Table 1: Drugs evaluated

\begin{tabular}{|c|c|c|c|c|c|}
\hline \multirow[b]{2}{*}{$\begin{array}{l}\text { Product } \\
\text { details }\end{array}$} & \multicolumn{5}{|c|}{ Product code } \\
\hline & $\mathbf{M}_{\mathrm{A}}$ & $M_{B}$ & $\mathrm{~T}_{\mathrm{c}}$ & $T_{D}$ & $\mathbf{S}$ \\
\hline $\begin{array}{l}\text { Generic } \\
\text { name }\end{array}$ & Metronidazole & Metronidazole & Tinidazole & Tinidazole & Secnidazole \\
\hline $\begin{array}{l}\text { Brand name \& } \\
\text { strength }\end{array}$ & $\begin{array}{l}\text { Flagyl } \\
200 \mathrm{mg}\end{array}$ & $\begin{array}{l}\text { Emtrin } \\
\text { 200mg }\end{array}$ & $\begin{array}{l}\text { Fasigyn } \\
500 \mathrm{mg}\end{array}$ & $\begin{array}{l}\text { Amtiba } \\
500 \mathrm{mg}\end{array}$ & $\begin{array}{l}\text { Flagentyl } \\
500 \mathrm{mg}\end{array}$ \\
\hline Manufacturer & May \& Baker & Emzor & $\begin{array}{l}\text { May \& } \\
\text { Baker }\end{array}$ & Cadila & May \&Baker \\
\hline Batch no. & IP 129 & XA & C12 & BS 104 & U132 \\
\hline Manuf./ Expiry & 02-2005/ & 03-2007/ & $12-2007 /$ & $11-2008 /$ & 09-2008/ \\
\hline date & $01-2010$ & 03-2010 & $12-2011$ & $10-2011$ & 09- 2012 \\
\hline $\begin{array}{l}\text { Dosage } \\
\text { form }\end{array}$ & Tablet & Tablet & Tablet & Tablet & Tablet \\
\hline
\end{tabular}


millitres each of the reference and test solutions were spotted on the plates. The plates were developed, allowed to dry in air for $3 \mathrm{~min}$ and placed in an iodine tank for 5 min. TLC was performed in three laboratories in universities in Nigeria to accommodate possible variations in findings arising from differences in environmental conditions. The environmental conditions were $30{ }^{\circ} \mathrm{C} / 45$ $\% \mathrm{RH}$ (University of Uyo); $25{ }^{\circ} \mathrm{C} / 65 \% \mathrm{RH}$ (University of Calabar); and $35{ }^{\circ} \mathrm{C} / 27 \% \mathrm{RH}$ (University of Sokoto). Three chromatograms were run per brand of drug/sample in each laboratory and the mean $\mathrm{R}_{\mathrm{f}}$ taken.

\section{RESULTS}

The results of the colour reactions for the various product brands are outlined in Tables 2 - 4 along with $R_{f}$ values. The colours observed for the generic metronidazole, i.e., $M_{A}$ and $M_{B}$, were similar for each of the reagent/test employed. Nitric acid test was not substituent-specific as orange colour was produced irrespective of the generic type of the nitroimidazole used. Sodium nitrite and bismuth subnitrate distinguished between metronidazole and the other two products as it gave a different colour.

Table 2: Colour reaction tests of 5nitroimidazole products.

\begin{tabular}{|c|c|c|c|}
\hline $\begin{array}{l}\text { Branded } \\
\text { drugs }\end{array}$ & $\begin{array}{l}\text { Nitric } \\
\text { acid test }\end{array}$ & $\begin{array}{l}\text { Sodium } \\
\text { nitrite } \\
\text { test }\end{array}$ & $\begin{array}{l}\text { Bismuth } \\
\text { subnitrate } \\
\text { test }\end{array}$ \\
\hline $\mathrm{M}_{\mathrm{A}}$ & Orange & Brown & Brown \\
\hline$M_{B}$ & Orange & Brown & Brown \\
\hline $\mathrm{T}_{\mathrm{C}}$ & Orange & Red & Red \\
\hline$T_{D}$ & Orange & Red & Red \\
\hline $\mathrm{S}$ & Orange & Red & Red \\
\hline
\end{tabular}

$M, T$ and $S$ represent metronidazole, tinidazole and secnidazole, respectively, with $A$ and $B$ as brands of metronidazole, $C$ and $D$ brands of tinidazole.

\section{DISCUSSION}

\section{Colour reactions}

Concentrated nitric acid, when employed with concentrated sulphuric acid, is used to indicate the aromatic character of a substance. A yellow colour is usually produced when $10 \% \mathrm{w} / \mathrm{v}$ sodium hydroxide is added to a substance with aromatic character, turning orange with excess alkali. The aminoimidazole formed by the zinc/hydrochloric acid reaction during the preparation of the test solution was reconverted via oxidation to nitroimidazole by nitration. Hence, reaction with concentrated nitric acid gave the expected result due to the aromatic nature of 5-nitroimidazoles, as the addition of $40 \% \mathrm{w} / \mathrm{v}$ sodium hydroxide provided the excess alkali required to produce an orange colour which results from the formation of an aromatic nitro compound.

Table 3: Mean $R_{f}$ values of various brands of 5nitroimidazole products evaluated in three laboratories, using mobile phase $A(n=3)$.

\begin{tabular}{cccccc}
\hline Lab, & $\mathbf{M}_{\mathbf{A}}$ & $\mathbf{M}_{\mathbf{B}}$ & $\mathrm{T}_{\mathbf{C}}$ & $\mathrm{T}_{\mathrm{D}}$ & $\mathbf{S}$ \\
\hline 1 & 0.460 & 0.470 & 0.750 & 0.730 & 0.520 \\
2 & 0.420 & 0.460 & 0.750 & 0.740 & 0.530 \\
3 & 0.460 & 0.470 & 0.770 & 0.760 & 0.540 \\
Mean & 0.447 & 0.467 & 0.757 & 0.743 & 0.530 \\
S.D \pm & 0.023 & 0.006 & 0.012 & 0.015 & 0.010 \\
\hline
\end{tabular}

$M, T, S$ denote metronidazole, tinidazole and secnidazole, respectively, while 1, 2, 3 denote laboratories in University of Sokoto, University of Uyo and University of Calabar, respectively.

Table 4: Mean $R_{f}$ values of the various rands of 5-nitroimidazole drugs in three aboratories, using mobile phase $B(n=3)$

\begin{tabular}{cccccc}
\hline Lab. & $\mathbf{M}_{\mathbf{A}}$ & $\mathbf{M}_{\mathbf{B}}$ & $\mathbf{T}_{\mathbf{C}}$ & $\mathbf{T}_{\mathbf{D}}$ & $\mathbf{S}$ \\
\hline 1 & 0.680 & 0.680 & 0.540 & 0.550 & 0.700 \\
2 & 0.680 & 0.640 & 0.530 & 0.530 & 0.710 \\
3 & 0.680 & 0.650 & 0.520 & 0.530 & 0.720 \\
Mean & 0.680 & 0.657 & 0.530 & 0.537 & 0.710 \\
S.D \pm & 0.000 & 0.021 & 0.010 & 0.010 & 0.010 \\
\hline
\end{tabular}

M, T, S denote metronidazole, tinidazole and secnidazole, respectively, while 1, 2, 3 denote laboratories in University of Sokoto, University of Uyo and University of Calabar, respectively.

All the brands of 5-nitroimidazole tested in our experiments gave an orange colour in the nitric acid test (Table 1). A drug that could be fraudulently labelled as an anti-infective is 
paracetamol due to its low cost and mild antipyretic effect [21]. It has been noted that some labelled anti-infectives only reduce symptoms of illnesses for a while. The colour observed was brown for metronidazole brands, $M_{A}$ and $M_{B}$, in sodium nitrite and bismuth subnitrate tests. Concentrated sulphuric acid reduced the nitro compound to the amino derivative as the sodium nitrite subsequently formed a diazotization derivative with the observed colour based on the extent of conjugation. Sodium nitrite in acidic medium forms diazonium salts with compounds bearing free amino group. The alkyl substituent on the imidazolyl nitrogen distinguishes the 5-nitroimidazole by the characteristic colours observed in sodium nitrate and bismuth subnitrate tests. The alkyl substitution on metronidazole is ethyl alcohol (a primary alcohol) which confers a brown colouration on the compound while tinidazole which bears ethyl sulphone gives a red colouration with the reagents. The $\mathrm{N}$ substitution on secnidazole is a secondary alcohol and this confers a red colour deeper in intensity than observed for tinidazole. The attached substituents on any system containing delocalized electrons either withdraw from the ring or donate electrons to it to a more or lesser extent. The colours, therefore, produced by such compounds depends on the availability and density of available electrons which affect the absorption and transmission of light.

\section{TLC Methods A and B}

The mobile phases $A$ and $B$ were designed to make a distinction between the 5nitromidazole compounds. In the TLC systems, metronidazole and secnidazole were differentiated from tinidazole by reason of their $R_{f}$ values. The $R_{f}$ values of metronidazole brands, $M_{A}$ and $M_{B}$ in the various laboratories were similar. A similar finding was observed for the brands of tinidazole, $T_{A}$ and $T_{B}$. The $R_{f}$ values of the brands of tinidazole were higher than those of metronidazole and secnidazole in the solvent systems (seeTable 4). This is probably due to its ethylsulphone substitution as against the primary and secondary alcohol functional group affiliations of metronidazole and secnidazole, respectively.

The spots produced with solvent system B were more conspicuous on exposure to iodine vapour in the tank. The movement of the solutes was also freer as indicated by $R_{f}$ values. While the spots for the brands of metronidazole and secnidazole were distinct, the ones for tinidazole exhibited tailing and had a lower $R_{f}$ (Table 4).

\section{Influence of some variables on the method}

The TLC experiments were conducted in laboratories situated in the three major geographic regions of Nigeria spanninging 25 - $60 \%$ relative humidity and a temperature range of $25-35{ }^{\circ} \mathrm{C}$. These tests were performed in different regions in order to determine if differences in temperature and humidity across the country would influence the tests. Although $R_{f}$ values were not highly reproducible, the relative pattern of appearance of the spots in the chromatograms was constant and, therefore, useful for drug identification.

\section{Identification system}

An identification system for the detection and screening of 5-nitroimidazole preparation was established by combining both the colour reaction and TLC tests. The identification system was rapid (Fig 2) and thus suitable for screening the 5 - nitroimidazole drugs tested in this work. However, since fraudulent labelling and other substandard features (e.g., excess or shortfall in the content of active ingredient in the formulation) often characterise unacceptable products, this identification system does not in any way obviate the need for quantitative evaluation of this group of drugs. 


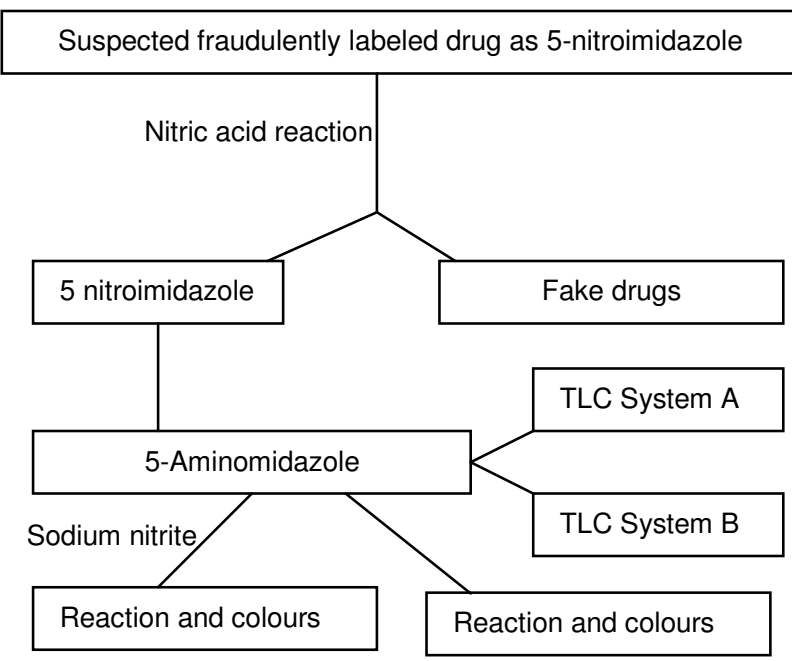

Fig 2: Identification tests for the detection of suspected fraudulently-labeled 5-nitroimidazole drugs

\section{CONCLUSION}

The developed identification system based on colours produced by chemical interaction of reagents with the functional groups present in the molecule of 5 - nitroimidazole drugs, and TLC methods can be jointly used to identify fake drugs that are fraudulently labelled as 5-nitroimidazole. This identification system should be useful to those involved in drug distribution, especially community pharmacies and pharmacy departments of hospitals since it will afford them a qualitative assessment of the products. It should, however, be emphasized that the tests evaluated do not eliminate the need for quantitative assessment of this group of drugs as may be necessary.

\section{ACKNOWLEDGMENT}

The authors are grateful to JDJIDE Pharmacy, Ikot Ekpene, Nigeria for financial support.

\section{REFERENCES}

1. Osibo O. Spurious Drugs - reading between the lines, Lanpharm. Scient. Lab. Services, Lagos. 1990; pp 1-4
2. World Health Organization. Guidelines for the inspection of drug distribution channels, WHO/PHARM.95.580 rev1, Geneva, Switzerland : WHO, 1997.

3.Tomlinson R. China crackdown on counterfeit medicines. BMJ 1999; 318: 624.

4. World Health Organization. Simple tests for drugs included in the WHO Model List of Essential Drugs, WHO/PHARM/95.583. /rev.1, Geneva, Switzerland: WHO 1994.

5. Ministry of Health and Welfare, International Affairs Division, Research and Development for Rapid Examination for Fake Drugs, Pharm./96.341, Japan. 1995.

6. National Agency for Food and Drug Administration and Control Act Cap N1. Laws of the Federation of Nigeria. 2004 .

7. World Health Organization Drug Information. Countering the counterfeiters. 1; 4; 195-196. WHO 1987.

8. Japanese Pharmacopoeia, Society of Japanese Pharmacopoeia, 13th ed., Shibuya, Tokyo. 1998.

9. British Pharmacopoeia. Vol. 2. The Pharmaceutical Press, Her Majesty's Stationery Office, London. 2003.

10 Yian $Q M$. Crackdown targets counterfeit drugs. Washington Post; Friday, November 20: 2009; p 5.

11. Liu HH, Dasgupta PK. Analytical chemistry in a drop solvent extraction in a microdrop. Anal. Chem.1996; 68: 1817-1821.

12. Gorski ED. Management of giardiasis, Am Fam Physician. 1985; 32:157.

13 Gray MJ, Slonecker PJ, Dennis $P$, Shalliker RA. J Chromatogr. 2005; A1096; 92-100.

14. Burnackis TG. Topical metronidazole for decubitis ulcers. Hospital Pharmacy.1989; 24: 960-961. 
Awofisayo et al

15. Hardman J, Limbird L, Gilman A. The Pharmacological Basis of Therapeutics, $8^{\text {th }}$ ed, McGraw- Hill Inc., New York, 2001;pp 14841485.

16. Jokippi L, Jokippi AMM. Single dose metronidazole and tinidazole as therapy for giardiasis: success rates, side effects and drug absorption and elimination. J. Infect. Dis.1979; (6): $140-184$

17. Moody M. A topical antimicrobial agent for malodorous wounds. $\mathrm{Br} J$ Nursing. 1998; 7: 286-289.
18. European Pharmacopoeia, 3rd ed., Council of Europe, Stuttgart, 1997; pp 1465-1539.

19. Giroud $C$, Ausburger M, Menetry A, Mangin PJ. Chromatogr.2003; B789: 131-138.

20. World Health Organization. Drug Information, 11; 2: 57-58, Geneva, Switzerland. WHO 1997.

21. Green MD, Dwight $L M$, Wirtz RA, White NJ. A colorimetric field method to assess the authenticity of drugs sold as the antimalarial artesunate. J.Pharm Biomed Anal.2000; 24: 65-70. 